\title{
The Extraction of Information from a Radar Display
}

The figure illustrating R. J. Turner's article in the last number $(27,533)$ was printed without its legend. A short summary of the paper and the illustration and legend follow.

The use and interpretation of a radar display requires a consideration of motion relative to different frames of reference. It is essential for sea or ground stabilization that the correct data is fed to the radar: Fig. I illustrates the velocity of the ship relative to different frames of reference and also the quantities measured by different types of log. Ideally, when a radar installation is being planned, the type of log to be installed should be chosen so that the radar can give the information required. With existing installations the type of log fitted should be borne in mind in deciding just what information the display is capable of giving.

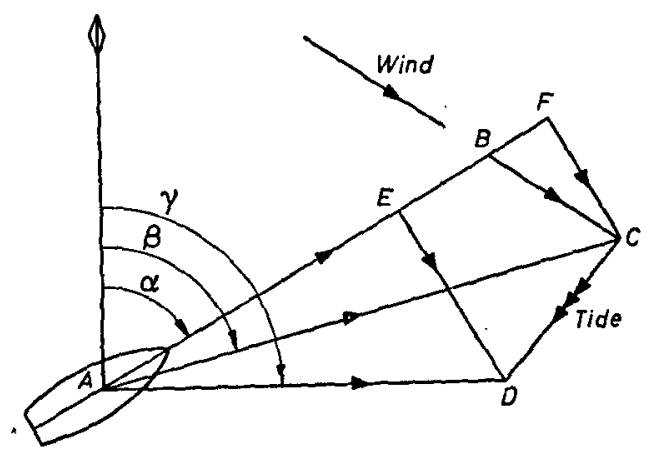

FIG. I

$\mathbf{A B}=$ velocity relative to the water with no wind

$\alpha=$ heading (measured by a compass)

$\mathbf{A C}=$ velocity relative to the water with a wind

$\beta=$ 'wake course'

$(\beta-\alpha)=$ 'leeway'

$\mathrm{AC}=$ speed relative to the water (measured by a towed log or a 2 -axis doppler log on sea returns)

$A F=$ fore and aft component of $A C$ (measured by an (i) electromagnetic log, (ii) single axis doppler log on sea returns, (iii) pressure/impeller $\log$ ). Note : for leeway of $8^{\circ} \mathrm{AF}$ differs from $\mathrm{AC}$ by $\mathrm{I} \%$

$A D=$ velocity relative to the ground

$\gamma=$ 'course made good'

$\mathrm{AD}=$ speed relative to the ground (measured by a 2 -axis doppler log on bottom returns)

$A E=$ fore and aft component of ground speed (measured by a single axis doppler log on bottom returns) 